\title{
NOUVELLE
}

\section{Des kératines du cheveu dans l'émail dentaire}

\section{Le lien entre maladies capillaires et caries}

Olivier Duverger
Laboratory of skin biology,

National institute of arthritis and musculoskeletal and skin diseases, National institutes of health, 50 South Drive, Bethesda MD 20892, États-Unis. duvergero@mail.nih.gov
Le poil et la dent :

\section{des appendices ectodermiques}

Au cours de l'embryogenèse, les appendices ectodermiques tels que le poil (ou le cheveu) et la dent se forment par une interaction complexe entre l'ectoderme superficiel et le mésoderme sous-jacent (Figure 1). Les étapes initiales de la morphogenèse du poil et de la dent sont très similaires morphologiquement et mettent en œuvre des signaux moléculaires communs échangés entre l'ectoderme et le mésoderme [1, 2]. La structure et la composition finales du poil et de la dent sont cependant très différentes. Les parties rigides du poil et du cheveu, qui se développent essentiellement à partir du composant ectodermique du follicule pileux, sont principalement constituées de kératines, protéines structurales fibreuses et hautement insolubles, qui leur confèrent une extrême résistance. Certaines des kératines du follicule pileux sont intégrées dans le poil lui-même et sont appelées kératines dures. D'autres sont présentes dans le tissu de soutien, qui forme une gaine autour du poil durant sa formation (gaine épithéliale interne et couche compagnon) et assure son ancrage dans la peau (Figure 1). La dent est formée de deux structures rigides majeures: la dentine, qui est produite par les odontoblastes (dérivé mésenchymateux), et l'émail, qui est produit par les améloblastes (dérivé épithélial) et recouvre la surface exposée de la dent (Figure I). L'émail dentaire est la substance la plus dure produite par l'organisme, avec $96 \%$ de minéraux. Au cours de sa formation, l'émail est constitué de protéines matricielles spécifiques (amélogénine, améloblastine, énaméline) déposées de façon très organisée et ensuite progressivement dégradées et remplacées par des cristaux d'hydroxyapatite. L'émail mature est formé de structures élémentaires juxtaposées, appelées prismes d'émail. Chacun de ces prismes est entouré d'une gaine de nature organique extrêmement insoluble, dont la nature protéique est longtemps restée énigmatique (Figure 1).

\section{Des kératines du poil dans l'émail dentaire}

Dans une étude récente menée au NIH dans le laboratoire de Maria I. Morasso, nous avons découvert que des kératines exprimées spécifiquement dans le tissu de soutien entourant le poil étaient également exprimées par l'organe produisant l'émail [3]. D'autre part, nous avons montré que l'une de ces kératines, la kératine 75 , est intégrée dans l'émail où elle est localisée au niveau de la gaine des prismes. Des études antérieures avaient suggéré que la matière organique présente dans l'émail mature avait des propriétés similaires à celles des kératines, mais la nature exacte des protéines qui la constituent n'avait pas été élucidée [4]. D'autre part, il est intéressant de noter que, dans la littérature antérieure à la caractérisation des protéines matricielles de l'émail (amélogénine et autres), ce dernier était considéré comme une structure kératinisée (comme le poil et l'ongle) avec un degré extrême de minéralisation [5]. L'identification de kératines spécifiques de certaines couches du follicule pileux dans l'émail, dont la distribution autour des prismes d'émail est comparable à leur distribution autour de la tige pilaire, prouve que cette vision n'était pas totalement erronée.

Mutations dans les kératines du poil et risque de caries

Deux polymorphismes dans le gène codant pour la kératine 75 ont été associés à deux maladies cutanées très distinctes. L'un est associé à la pseudofolliculite de la barbe (pseudo folliculitis barbae), dans laquelle les extrémités de poils rasés de près ont tendance à se recourber et à pénétrer dans la peau, entraînant la formation de papules folliculaires inflammatoires non microbiennes [6]. Cette condition est plus fréquente chez les mâles de race noire, en raison de la nature courbe de leurs poils. Le second polymorphisme est associé au syndrome du cheveu anagène lâche (loose anagen hair syndrome) qui affecte des enfants dont les cheveux peuvent être facilement arrachés [7]. Ces deux symptomatologies démontrent clairement la fonction cruciale de la kératine 75 dans la gaine de soutien entourant le poil, assurant son guidage au cours de sa croissance ainsi que son ancrage dans la peau. Grâce à une collaboration avec les départements de génétique et d'odontologie de l'université de Pittsburgh (Pennsylvanie, États-Unis), permettant d'associer données génétiques et examens intra-oraux dans une large population d'adultes et d'enfants, nous avons montré que ces deux polymorphismes dans la kératine 75 étaient également associés à un risque plus élevé de développer des caries dentaires [3]. 

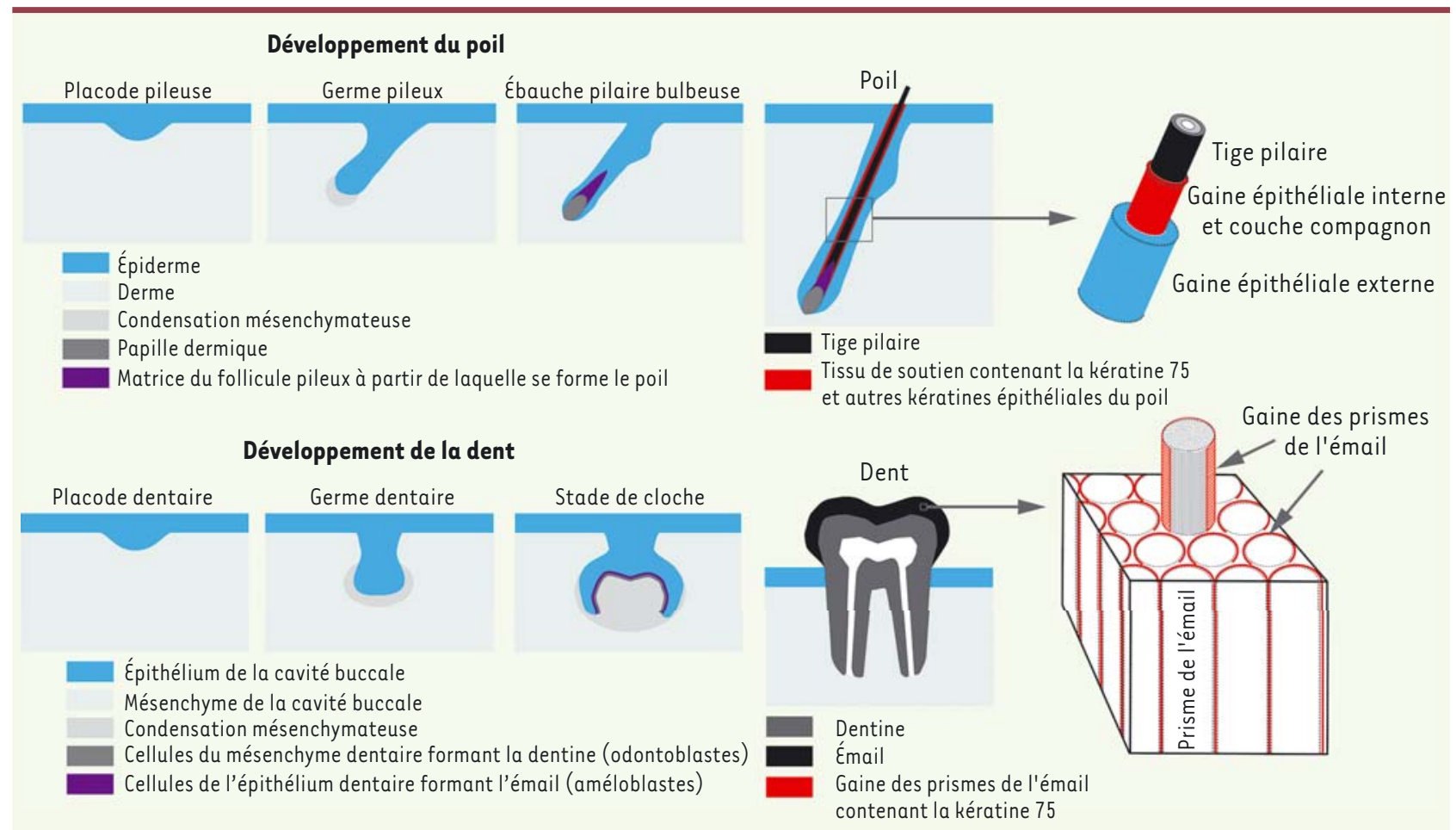

Figure 1. Développement du poil et de la dent, et expression de la kératine 75 dans le follicule pileux et l'émail dentaire. Les différentes étapes du développement du poil et de la dent à partir d'une interaction entre ectoderme et mésoderme sont indiquées. Les structures finales dérivées du compartiment ectodermique de l'appendice, en l'occurrence la tige pilaire et l'émail dentaire, sont représentées en noir. La localisation des protéines épithéliales du poil dans le follicule pileux et dans l'émail est indiquée en rouge. Dans le follicule pileux, la kératine 75 est présente spécifiquement dans la couche compagnon séparant les gaines épithéliales interne et externe. D’autres kératines du poil, présentes dans la gaine épithéliale interne, sont également exprimées par l'organe de l'émail.

Fonction des kératines du poil dans

\section{l'émail dentaire}

En analysant de près l'émail de patients porteurs du polymorphisme associé à la pseudo folliculite de la barbe, nous avons démontré que la mutation dans la kératine 75 rend la gaine des prismes de l'émail instable, affecte l'arrangement des prismes, est associée à une réduction de la dureté de la couche interne de l'émail, et favorise la formation de lésions carieuses tubulaires [3]. De façon similaire à sa fonction dans le follicule pileux, la kératine 75 semble donc jouer un rôle crucial dans le guidage des prismes de l'émail au cours de leur formation ainsi que dans le maintien de leur cohésion dans l'émail mature. D'autre part, il a été montré que l'attaque acide des caries débute au niveau de la gaine des prismes de l'émail et progresse vers les parties plus minéralisées. La stabilité des protéines constituant la gaine des prismes joue un rôle dans la résistance aux caries $[8,9]$. II semblerait donc que la kératine 75 et d'autres kératines du poil présentes dans l'émail contribuent, via leurs propriétés biochimiques uniques, à la stabilisation de la gaine de l'émail. Les mutations dans la kératine 75 déstabilisent cette gaine de nature organique entourant les prismes de l'émail, ce qui favorise ainsi l'installation et la progression de caries.

\section{Impact médical}

La carie dentaire est de loin la maladie infectieuse chronique la plus répandue, affectant toutes les tranches d'âge. Le développement des caries fait intervenir de nombreux facteurs tels que l'alimentation, le mode de vie, l'hygiène, mais aussi des facteurs génétiques affectant la structure de l'émail ou la composition de la salive [10]. Ainsi, connaître les marqueurs génétiques associés à un risque plus élevé de développer des caries peut permettre une approche préventive plus efficace. Ceci est particulièrement vrai lorsque ces marqueurs génétiques se manifestent par des signes extérieurs visibles, comme dans le cas de la pseudo folliculite de la barbe. Cette étude souligne ainsi l'importance de la collaboration entre différentes spécialités médicales telles que la dermatologie et l'odontologie. $\diamond$

Hair keratins in tooth enamel: the link between hair disorders and dental caries

\section{LIENS D'INTÉRÊT}

L'auteur déclare n'avoir aucun lien d'intérêt concernant les données publiées dans cet article. 


\section{RÉFÉRENCES}

1. Pispa J, Thesleff I. Mechanisms of ectodermal organogenesis. Dev Biol $2003 ; 262$ : 195-205.

2. Biggs LC, Mikkola ML. Early inductive events in ectodermal appendage morphogenesis. Semin Cell Dev Biol 2014 ; 25-26 : 11-21.

3. Duverger 0 , Ohara T, Shaffer JR, et al. Hair keratin mutations in tooth enamel increase dental decay risk. J Clin Invest 2014 ; 124 : 5219-24.
4. Robinson C, Shore RC, Kirkham J. Tuft protein: its relationship with the keratins and the developing enamel matrix. Calcif Tissue Int 1989 ; 44 : 393-8.

5. Pautard FG. Mineralization of keratin and its comparison with the enamel matrix. Nature $1963 ; 199$ : 531-5.

6. Winter $\mathrm{H}$, Schissel D, Parry DA, et al. An unusual Alal2Thr polymorphism in the $1 \mathrm{~A}$ alpha-helical segment of the companion layer-specific keratin K6hf: evidence for a risk factor in the etiology of the common hair disorder pseudofolliculitis barbae. J Invest Dermatol $2004 ; 122: 652-7$.

\title{
NOUVELLE
}

\section{Traitement de la protéinose alvéolaire par transplantation intrapulmonaire de macrophages}

\author{
Raphael Borie $e^{1-3}$, Claire Danel ${ }^{3,4}$, Catherine Lainé ${ }^{5}$, \\ Caroline Kannengiesser ${ }^{3,6}$, Bruno Crestani ${ }^{1-3}$
}

La protéinose alvéolaire pulmonaire

La protéinose alvéolaire pulmonaire (PAP) est une maladie rare caractérisée par une accumulation de matériel phospholipoprotéinacé (protéines et lipides du surfactant) dans les alvéoles pulmonaires. Cette accumulation est liée à un défaut de clairance par les macrophages alvéolaires. Le diagnostic de protéinose alvéolaire pulmonaire est suggéré par un scanner thoracique évocateur (Figure I), et confirmé par une lecture spécifique du lavage bronchoalvéolaire [1, 2].

On distingue trois types de protéinose alvéolaire pulmonaire en fonction de leur étiologie: auto-immunes, secondaires et génétiques. Chez l'adulte, les formes auto-immunes, avec présence d'auto-anticorps sériques anti GM-CSF (granulocyte-macrophage colonystimulating factor) sont les plus fréquentes. Les formes secondaires sont dues le plus souvent à une inhalation de toxiques ou à une dysfonction du macrophage alvéolaire, en rapport soit avec une maladie hématologique, soit avec un déficit immunitaire. On ne détecte pas d'anticorps anti GM-CSF dans ces formes.

Les protéinoses alvéolaires pulmonaires d'origine génétique peuvent s’intégrer dans des syndromes touchant plusieurs organes, elles s'observent essentiellement chez l'enfant et leur présentation radio-clinique est relativement spécifique du gène en cause.

Les symptômes sont peu spécifiques et se limitent le plus souvent à une dyspnée. Il existe un risque d'infection opportuniste au cours des protéinoses alvéolaires pulmonaires auto-immunes, et la présence d'anticorps anti-GM-CSF pourrait être associée à un risque spécifique de méningite à cryptocoque [3] .

\section{Physiopathologie}

Le surfactant est composé d'un mélange de protéines et de lipides (essentiellement de la phosphatidylcholine) sécrétés par les pneumocytes de type II. La clairance du surfactant est réalisée par les pneumocytes de type II et les macrophages alvéolaires [4]. Le surfactant réduit la tension de surface alvéolaire et empêche le collapsus alvéolaire au cours
7. Chapalain V, Winter H, Langbein L, et al. Is the loose anagen hair syndrome a keratin disorder? A clinical and molecular study. Arch Dermatol 2002 ; 138 : 501-6.

8. Pincus P. Relation of enamel protein to dental caries. Nature 1948 ; 161 : 1014.

9. Little K. Caries-prone and caries-resistant teeth. Nature $1962 ; 193$ : 388-9.

10. Shaw JH. Causes and control of dental caries. N Engl J Med 1987 ; 317 : 996-1004.
${ }^{1}$ APHP, hôpital Bichat, DHU Fire, service de pneumologie $A$, centre de compétence des maladies pulmonaires rares, 46 , rue Henri Huchard, 75018 Paris, France;

2 Inserm unité 1152, Paris, France ; ${ }^{3}$ Université Paris Diderot, Paris, France ; ${ }^{4}$ APHP, hôpital Bichat, service d'anatomopathologie, 46, rue Henri Huchard, 75018 Paris, France ;

${ }^{5}$ service d'immunologie-thérapie cellulaire et hématopoïèse, centre hospitalo-universitaire Pontchaillou, Rennes, France;

${ }^{6}$ APHP, hôpital Bichat, service de génétique, 46, rue Henri Huchard, 75018 Paris, France. raphael.borie@bch.aphp.fr

du cycle ventilatoire. II intervient aussi dans la régulation de la réponse antiinfectieuse alvéolaire. L'insuffisance de surfactant chez les nouveau-nés prématurés (maladie des membranes hyalines), ou son altération au cours du syndrome de détresse respiratoire aiguë, sont ainsi responsables d'atélectasies conduisant à l'insuffisance respiratoire. Le GM-CSF joue un rôle capital dans la physiopathologie de la protéinose alvéolaire pulmonaire. Ce facteur de croissance des lignées granuleuses et monocytaires stimule in vitro la différenciation, la prolifération et la survie des cellules myéloïdes: monocytes, macrophages, éosinophiles, neutrophiles et cellules dendritiques. Le développement des souris mutantes déficientes (knockout) pour le gène codant pour le GM-CSF a permis de manière inattendue d'identifier la responsabilité de cette cytokine dans la physiopathologie de la maladie [5]. Ces souris développent une protéi- 